\title{
Information Fusion Method for Building Grassroots Teaching Organization Based on Intelligent Optimization
}

\author{
Ying Zhao \\ Guilin Tourism University, Guilin, Guangxi 541006, China \\ Correspondence should be addressed to Ying Zhao; zhaoying@gltu.edu.cn
}

Received 20 December 2021; Revised 13 January 2022; Accepted 28 January 2022; Published 3 March 2022

Academic Editor: Zhiguo Qu

Copyright () 2022 Ying Zhao. This is an open access article distributed under the Creative Commons Attribution License, which permits unrestricted use, distribution, and reproduction in any medium, provided the original work is properly cited.

\begin{abstract}
At present, the existing teaching construction information fusion methods are based on the classification results of teaching resources, but ignoring the global intelligent optimization of the fusion results, which leads to the deviation of the grassroots teaching organization construction information fusion results, and the fusion index value is low. Therefore, the information fusion method of the grassroots teaching organization construction based on intelligent optimization is proposed. The basic framework of information fusion is designed, and the information fusion is divided into the data layer, characteristic layer, and decision layer. The information characteristics of the construction of grassroots teaching organizations were extracted. The particle swarm optimization algorithm is used to search the maximum value of the work entropy of teaching grassroots unit, and the threshold value of teacher construction and grassroots unit work is used to realize the information fusion of teaching grassroots unit construction. Experimental results show that the proposed method has higher error rate, stability, efficiency index, and higher information fusion value and accuracy than the traditional method.
\end{abstract}

\section{Introduction}

Grassroots teaching organizations in institutions of higher learning are teaching organizations set up according to courses, majors, or subjects, grassroots organizations that directly organize teaching and carry out teaching research in accordance with their own teaching work plans under the direct leadership of colleges and other teaching organizations and are the most basic organizations of school teaching organization, teaching management, and teaching reform [1]. Grassroots teaching organization is the most basic teaching organization in colleges and universities to implement teaching tasks, promote teachers' teaching development, organize academic research, and undertake group teaching activities, which directly affect the education and teaching quality of colleges and universities. Grassroots teaching organizations in a broad sense include departments, teaching departments, teaching teams, teaching bases, teaching and research rooms, teaching and research groups, course groups, and experimental teaching centers; in a narrow sense, it usually refers to traditional teaching and research rooms or course groups [2]. However, due to the diversity of teaching organization information, its processing is difficult. In order to optimize the construction of grassroots teaching organizations in colleges and universities, some better information fusion methods have been put forward in current related fields.

Reference [3] proposes a design method of multimedia integrated learning resource information integration system in cloud storage environment based on adaptive balanced allocation and fuzzy scheduling. For the collected multimedia integrated learning resource information, the association rule mining method is used for attribute configuration, the resource configuration and information clustering are carried out according to the attribute classification results of multimedia learning resources, the information fusion model of resource integration is constructed, and the fuzzy scheduling method is used to realize the optimal integration of multimedia integrated learning resource information. The resource integration system software is developed and designed in the embedded environment, and the ZigBee protocol is used for the networking design and modular development of the multimedia integrated learning resource information integration system. Reference [4] puts forward 
the design scheme of the intelligent classroom platform based on artificial intelligence. The smart classroom platform adopts the intelligent design scheme of remote automatic control and bus integrated control, uses the lower computer integrated information processing method for sensor information processing, carries out bus transmission and information fusion of multimedia teaching information through the integrated information processing terminal, and carries out intelligent communication design in the upper computer module. Combined with decentralized control, the cross-compilation operation is carried out to realize real-time transmission and distributed storage of classroom platform control instructions and realize the optimal design of smart classroom platform in embedded environment

The above teaching construction information fusion method is to allocate resources and cluster information according to the attribute classification results of teaching resources and construct the information fusion model of resource integration but ignores the global intelligent optimization of the fusion results, resulting in the unsatisfactory information fusion results of the construction of grassroots teaching organizations. Therefore, an information fusion method of the grassroots teaching organization construction based on intelligent optimization is proposed.

\section{Current Situation and Characteristics of Grassroots Teaching Organization Construction}

2.1. Analysis of Current Situation and Problems. The grassroots teaching organizations in colleges and universities in China have played an important role in teaching management, specialty construction, curriculum and teaching material construction, teaching staff construction, teaching research, and teaching reform and achieved remarkable progress and results. However, with the expansion of the scale of colleges and universities, the problems of grassroots teaching organizations gradually appear.

\section{(1) The function of teaching organization is ignored}

Teaching and scientific research should complement each other. The combination of the two can better help college teachers organize and implement teaching work more effectively and reasonably. However, in fact, teaching and research achievements lack unified academic quality evaluation standards and academic quality assurance system corresponding to their achievements. Scientific research has not completely become an important driving force to improve the teaching level and education quality of contemporary college teachers but has an institutional impact on the development of grassroots teaching organizations in colleges and universities.

Since the reform and opening up, the fit between the grassroots teaching organization (Teaching and Research Office) system and China's higher education administration system has been declining day by day. On the one hand, in the era of scientific research-oriented administration, the administrative responsibilities of grassroots teaching organi- zations such as teaching and research offices in colleges and universities have passively expanded from single teaching and research to many administrative aspects such as scientific research and teacher team construction. However, the reform of supporting structure and system lags behind, and the grassroots teaching organizations such as teaching and research offices have been overloaded for a long time. The orientation is gradually blurred, the function is continuously weakened, and the operation and development are seriously hindered. On the other hand, due to the serious lack of its business learning and teaching discussion mechanism [5], grassroots teaching organizations such as teaching and research offices in colleges and universities are in vain and lack of construction, and the failure of positive function gradually moves towards the edge.

(2) Weakening of teaching research and reform activities

There are some problems in grassroots teaching organizations such as teaching and research offices of colleges and universities, such as the weakening of the management system of collective teaching and research function and the institutionalization of activity organization and management. Some grassroots teaching organizations such as teaching and research departments in colleges and universities mainly undertake the management of classroom teaching and the training of teachers and can well complete the basic tasks such as the organization, implementation, and assessment of classroom teaching. However, for teacher training, the degree of attention paid to raising is not enough, and it is not well implemented. At the same time, under the background of interdisciplinary integration and compound discipline talent training in colleges and universities, some grassroots teaching organizations such as teaching and research offices in colleges and universities can only carry out teaching and research activities in a certain discipline or even a certain course, and their teaching function needs to be further expanded.

The normal development of collective teaching and research activities in colleges and universities is often an effective way to mobilize and promote all teachers to communicate with each other and improve their comprehensive quality. At present, many grassroots teaching organizations such as teaching and research offices in colleges and universities do not pay enough attention to their collective teaching and research activities, lack of system construction, and organizational arrangements; teaching and research activities are difficult to carry out normally, and the quality of organizing teaching and research activities is not high.

The serious weakening of the function of grassroots teaching organizations and the serious lack of teaching and research activities make teachers' teaching lose their professional support, and teaching activities are in a random state, which is not conducive to improving teaching quality.

Teachers' teaching activities lack a way and platform to directly improve their teaching practice ability. Teachers and teams lose a sense of collective belonging and responsibility to grassroots teaching organizations such as teaching 
and research offices in colleges and universities, which is not conducive to teachers' personal development and effective team construction [6].

\section{(3) The training of young teachers lags behind}

In reality, due to the large span between disciplines and majors, teachers often lack effective channels and platforms for teaching theory communication and skill exchange. Even within the grassroots teaching organizations of the same major, due to the differences of courses, the communication and discussion among teachers often focus on the general level of the teaching process, such as the formulation of teaching calendar, courseware making, and other basic teaching activities. They lack understanding and communication about the problems and difficulties that may be encountered in course teaching, let alone joint discussion and cooperation. Some teachers tend to teach individually with little mutual cooperation, many effective teaching methods and experience can not be inherited and shared, and there is a lack of communication, help, and guidance between new and old teachers. Young teachers often lack the overall understanding and grasp of the professional curriculum system and talent training scheme and do not have a good understanding of the intersection and inheritance of knowledge points between courses. In the process of actual teaching, it leads to frequent situations of seeking perfection and regardless of primary and secondary and can not achieve the teaching effect of highlighting key points and understanding in simple terms. Although there is a perfect theoretical training system for talents, due to the inadequate implementation of teaching division of labor, the repetition, and even contradiction of teaching process, the curriculum teaching of the whole specialty is disjointed and unsystematic. The above problems are closely related to the lag of grassroots teaching organizations in training young teachers and weak team construction, which makes it difficult to significantly improve the teaching effect and teaching quality, and the overall level of professional construction will eventually be seriously affected [7].

\section{(4) Lack of teacher teaching community}

The grassroots teaching organizations in colleges and universities, especially the teaching and research offices in the traditional sense, are essentially teachers' teaching communities. For the teaching and Research Office, the purpose of its setting is mainly to enable similar courses and professional teachers to implement cooperation and achieve the goal of talent training. However, the current situation is that although the teachers in each teaching and research department become a member of the organization in form, their actual powers related to their vital interests, such as salary assessment performance, professional title qualification evaluation, and subject research project application, are concentrated in secondary colleges, and the teaching and research work is a mere formality. Teachers' teaching tasks are usually divided into their own. In the process of teaching, teachers gather less and leave more, and communication is insufficient. In addition, the internal and external interaction of teaching and research is also relatively poor. Teaching and research exchange activities are not often carried out within the organization, the construction of curriculum teaching system and teaching research are sometimes absent, and the observation, listening, or evaluation of courses in the teaching process is a mere formality, lacking a strong teaching and research atmosphere. The teaching and Research Office also has a strong closeness and rarely carries out academic exchanges with the outside world, which is not conducive to the broadening of teachers' thinking vision and the complementarity of academic information exchange, which seriously hinders the growth of high-level and compound teachers. On the whole, the discrete state of grassroots teaching organizations such as teaching and research offices in colleges and universities is obvious, the cohesion needs to be strengthened, and the community is seriously missing [8].

\subsection{Contents and Characteristics of Grassroots Teaching} Organization Construction. With the deepening of teaching reform in colleges and universities, the information needed to be managed by grassroots teaching organizations is increasing day by day, including the documents and materials with preservation and use value formed in the teaching management, teaching practice, and teaching research activities of various grassroots teaching organizations. It is the true record of talent training and management process, the crystallization of teachers' hard work, and the proof of the strength of grassroots teaching organization $[9,10]$. The construction of basic teaching organization mainly includes teaching management, scientific research management, personnel management, and information. Among them, teaching management includes the inquiry management of teaching materials, teaching tasks, and curriculum information; scientific research management includes the management of publication of scientific papers and scientific research projects; personnel management refers to the management of basic information of teachers; daily management includes the release of announcements to groups and the management of information transmission to specific personnel.

The information that the basic teaching organization needs to manage is extensive, including teacher management, scientific research management, and teaching management. It has its own characteristics.

\section{(1) The division of teaching administration is special}

As an important part of grassroots teaching organizations, teaching management is divided not by ordinary years but by teaching year and schooling year, that is, from September 1 of each year to August 31 of the next year as a teaching year. Therefore, the division of the content of teaching management information is based on the teaching year and the number of years the students enrolls and graduates. This is a distinguishing feature from other management information architectures. 
(2) High requirements for retrieval

As the basic organization of management teaching and scientific research, it has its own characteristics to all kinds of information inquiry and often needs multicondition inquiry. For example, a search query for the author of a scientific paper should not only search all the authors but also the ranking of the first author or each author. This requires careful consideration of the specific needs of each of the managed content.

\subsection{Construction Structure of Grassroots Teaching} Organization. The architecture adopts modular structure, and the division and design of each module comply with the principles of high cohesion and low coupling. Each subarchitecture module is led out from the main module, and then, independent functional modules are led out from each subarchitecture module for independent debugging, and then, the integration and debugging of the whole architecture are carried out. According to the link of information management in our office, the structure design can be divided into eight substructures:

(1) Teacher information management substructure: to achieve the management of the basic information of the organization's teachers

(2) Substructure of teaching material management: centralized management of teaching materials for courses undertaken by the organization, such as training programs, course standards, implementation plans, lesson plans, courseware, teaching materials, and test paper libraries

(3) Teaching task management substructure: realize the management of the teaching tasks undertaken by the organization over the years, such as teaching hours, semesters, training levels, classes, teaching staff, course names, course nature, and hours

(4) Curriculum inquiry management substructure: realize the management of the information of the current semester courses undertaken by the organization, such as training level, class schedule, instructor, course name, course nature, number of hours, teaching time, and teaching place

(5) Paper publication management substructure: to realize the management of the information on the publication of scientific and technological papers by the teachers of the organization, such as the author, title, name of the publication, publication time, retrieval, and award winning

(6) Scientific research project management substructure: realize the management of the information on the participation of faculty members in scientific research projects, such as project source, research cycle, research funds, responsible persons, participation, and project introduction, and at the same time, pay attention to the decryption of relevant information on confidential projects

(7) Announcement management substructure: achieve the management of announcements issued by all teachers of the organization

(8) Notification issuance management substructure: achieves the management of notifications sent by specific staff members of the organization

\section{Information Fusion Method of Grassroots Teaching Organization Construction Based on Intelligent Optimization}

3.1. Theoretical Description of Information Fusion Technology. Information fusion [11-13] is a comprehensive information processing technology that cooperatively uses multisource information to obtain a more objective and essential understanding of the same thing or target. Fusion refers to collecting and integrating various information sources to generate complete, accurate, timely, and effective comprehensive information. It is more concise, less redundant, and more useful than the information directly obtained from various information sources. The purpose of fusion is to integrate individual elements through specific combination rules and the best cooperation to obtain more useful information, so as to improve the confidence of information, reduce the fuzziness of reasoning, and improve the decision-making ability.

3.2. Integration Principle of Teacher Construction and Grassroots Work in the Construction of Grassroots Teaching Organizations. During the integration of teacher construction and grassroots work in the construction of grassroots teaching organizations, the eigenvalues of teacher construction matrix in the construction of grassroots teaching organizations are calculated by using the grassroots work data band. On this basis, the eigenvalues of teacher construction in the construction of grassroots teaching organizations are arranged in order from big to small, and the integration of teacher construction and grassroots work is realized by using the results. The specific steps are detailed below.

In the process of integrating teacher construction and grassroots work in the construction of grassroots teaching organizations, $M$ represents the data band of grassroots work and $u$ represents the eigenvalues of teacher construction matrix in the construction of grassroots teaching organizations $[14,15]$; then, the following formula is used to arrange the eigenvalues of teacher construction in the construction of grassroots teaching organizations:

$$
X=M \frac{Y}{u}
$$

In the above formula, $Y=\left[y_{1}, y_{2}, \cdots, y_{u}\right]$ represents the set of characteristic values of teacher construction in the grassroots teaching organization construction.

In the process of integration of teacher construction and grassroots work in the construction of grassroots teaching 


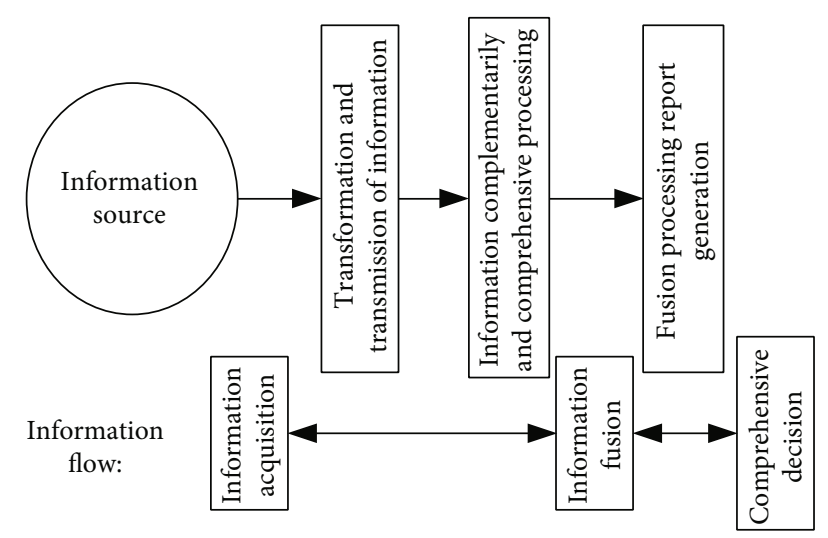

FIGURE 1: Basic framework of information fusion.

organizations, the following methods are used to integrate teacher construction and grassroots work:

$$
R=\mathfrak{\Im} \cdot \frac{u}{X}
$$

In the above formula, $\mathfrak{I}$ represents the integration coefficient of teacher construction and grassroots work. To sum up, it can be explained that the integration principle of teacher construction and grassroots work in the construction of grassroots teaching organizations has effectively completed the integration of teacher construction and grassroots work in the construction of grassroots teaching organizations.

3.3. Basic Architecture of Information Fusion. The four basic elements of information fusion architecture are as follows: information source, which can provide initial data; information transformation and transmission, which can realize information preprocessing; information complementarity, which is to complete information sublimation; and information fusion processing report, which can show the final result of fusion processing. The basic architecture diagram is shown in Figure 1.

\subsection{Hierarchical Classification of Information Fusion}

(1) Data level information fusion

Data layer fusion belongs to direct fusion of initial information of grassroots teaching at the same level, and comprehensive analysis of unprocessed grassroots teaching data is the lowest level of fusion [16]. The main advantages of this level of integration are as follows: it can keep as much as possible the construction of grassroots teaching organizations, access to other levels do not have the details. The downside is that processing is expensive and time consuming. The fusion process is illustrated in Figure 2:

(2) Feature level information fusion

The process of feature level fusion is as follows: Firstly, the feature is extracted from the initial grassroots teaching organization, and then, the feature information is analyzed and processed.

The merit of feature layer fusion is that it can compress the information and achieve real-time processing. The fusion result can embody the information characteristic of basic teaching organization. The fusion process is shown in Figure 3.

(3) Decision-level information fusion

Decision-level information fusion provides theoretical basis for detection, manipulation, and decision-making. It is necessary to consider from the perspective of demand, use the information of feature fusion to realize decision fusion, and its result directly affects the quality of decision.

The advantage of information fusion in the decision layer is that it has low cost, strong flexibility, and strong anti-interference performance, see Figure 4 for details.

As can be seen from Figure 4, the information fusion process of decision-making level is as follows: build information features of different grass-roots teaching organizations, then make decisions through feature fusion, fuse and summarize the information after decision-making, and output the final decision-making information.

3.5. Information Feature Extraction of Grassroots Teaching Organization Construction. The information perception process of the construction of grassroots teaching organizations is affected by the information characteristics and structure environment factors of each platform, and there will be some abnormal information or noise interference in the information. In order to ensure the effective information perception in the fusion process, the redundant information is eliminated through information feature extraction, and valuable feature information of each platform is obtained for fusion $[17,18]$.

Multivariate discriminant analysis [19] is a linear supervised feature extraction method. By maximizing class divergence and minimizing class divergence, class information distance is reduced, class information distance is increased, and projection direction of information samples is distinguished.

Let the platform information sample set be $W$, which is composed of $n$ subsets, and each subset belongs to $n$ different categories. The description formula of $W$ is given by

$$
W=\left\{w_{1}, w_{2}, \cdots, w_{n}\right\}
$$

The number of subset samples in this set is

$$
n=\sum_{i=1}^{u} n_{i}
$$

The intraclass divergence matrix of set $W$ is $R$, and the corresponding calculation formula is

$$
R=\sum_{i=1}^{u} \sum_{j=1}^{u}\left(R-w_{n}\right)^{T} .
$$




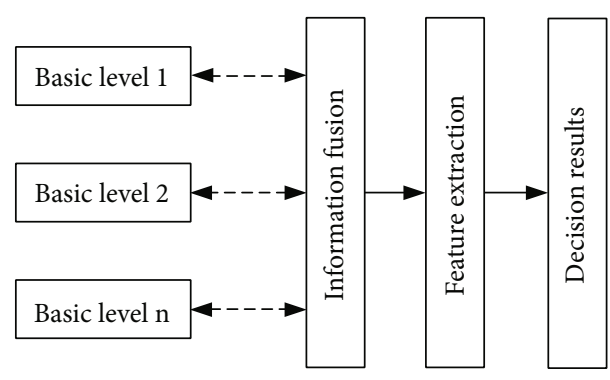

Figure 2: Schematic diagram of data layer fusion.

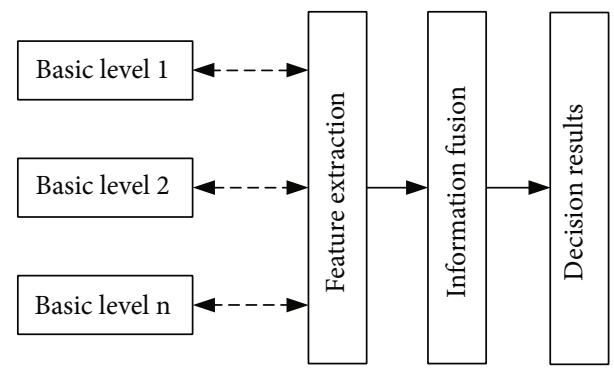

FIGURE 3: Schematic diagram of feature level information fusion.

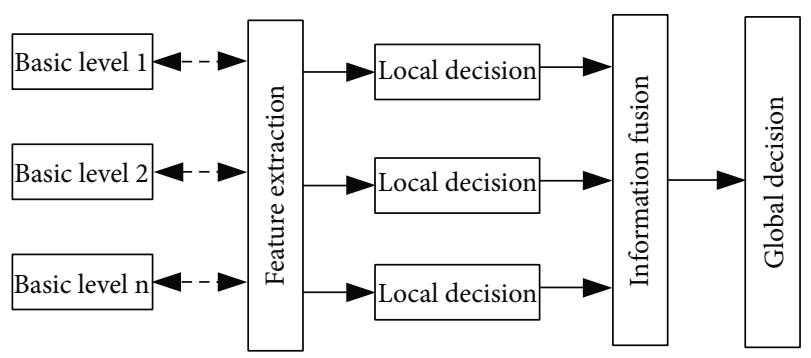

Figure 4: Schematic diagram of decision level information fusion.

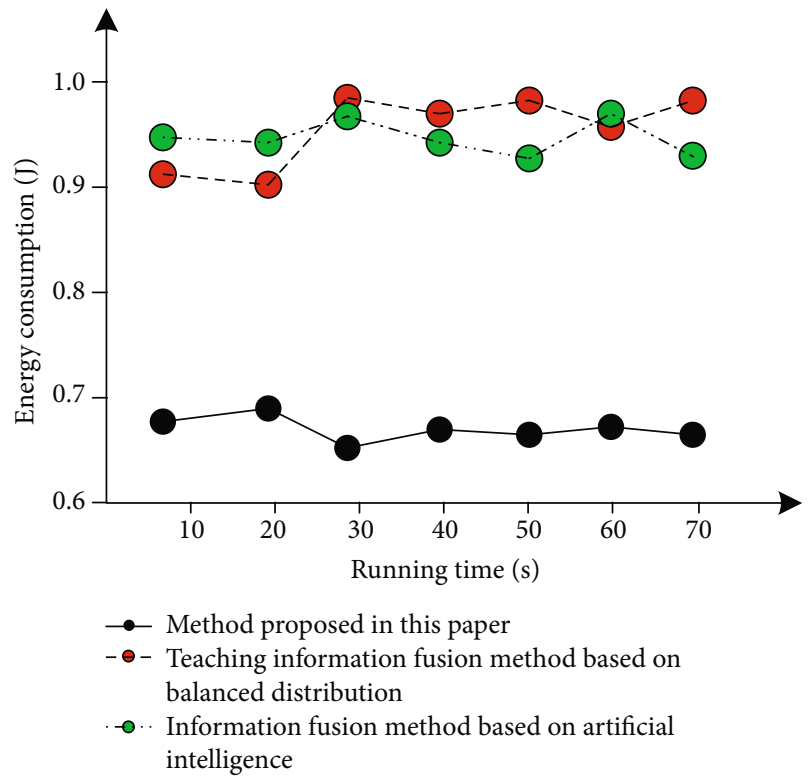

Figure 5: Comparison of energy consumption of different information fusion methods.

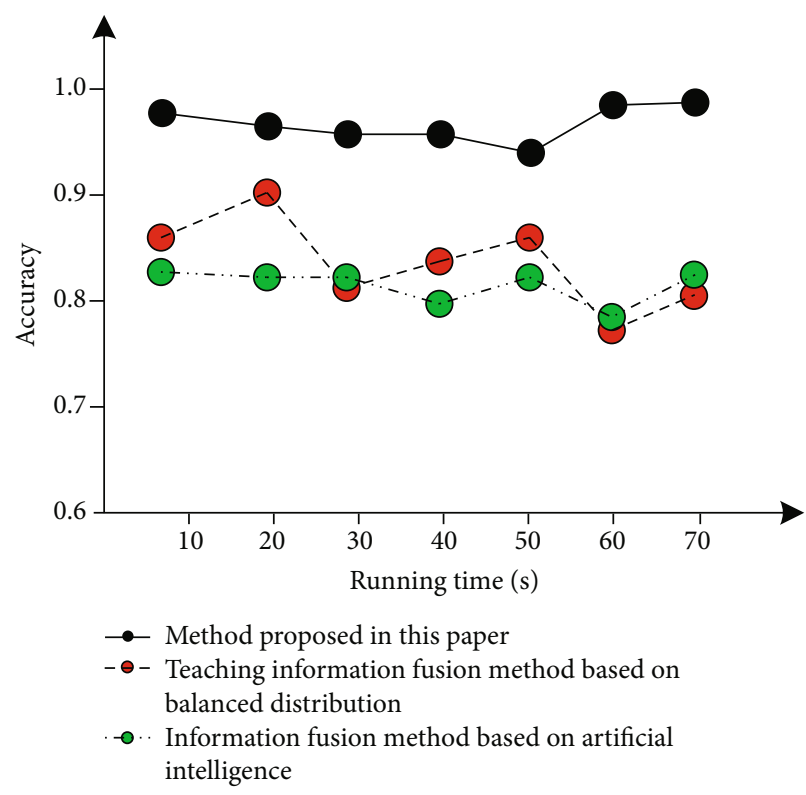

Figure 6: Accuracy comparison of different information fusion methods.

Eigenvector $Q=\left[q_{1}, q_{2}, \cdots, q_{d}\right]$ corresponding to the largest $d$ eigenvalue is the linear projection direction of the platform information, based on which the linear features are extracted and classified [20]. The objective functions and corresponding constraint conditions of the multivariate judgment analysis method are shown in

$$
f_{\mathrm{MAX}}=\max \frac{Q^{T} R Q}{Q^{T} R Q},
$$

$$
\text { s.t. } Q^{T} Q=1 \text {. }
$$

The above formula is called the generalized Rayleigh quotient. In order to obtain the maximum value of the objective function, the maximum eigenvalue is calculated by using the following formula:

$$
R Q=\delta f \cdot Q
$$

In the above expression, $\delta$ represents the maximum eigenvalue and $\delta$ corresponds to the eigenvector that maximizes the objective function.

By analyzing and integrating the local linear characteristics of platform information, the global nonlinear structural characteristics of platform information are obtained by using local tangent space permutation method [21]. According to the tangent space orthogonal basis corresponding to the local neighborhood of the sample point, the coordinates of the point in the local tangent space are calculated.

3.6. Information Fusion of Grassroots Teaching Organization Construction Based on Intelligent Optimization. In the process of optimizing and integrating teacher construction and grassroots work in the construction of grassroots teaching organizations, based on the abovementioned estimation 
TABLE 1: Comprehensive effectiveness of different information fusion methods.

\begin{tabular}{|c|c|c|c|c|c|c|c|c|c|}
\hline \multirow[t]{2}{*}{ Number of experiments } & \multicolumn{3}{|c|}{ Proposed method } & \multicolumn{3}{|c|}{$\begin{array}{l}\text { Teaching information fusion } \\
\text { method based on balanced } \\
\text { distribution }\end{array}$} & \multicolumn{3}{|c|}{$\begin{array}{l}\text { Information fusion method based } \\
\text { on artificial intelligence }\end{array}$} \\
\hline & Error rate & Stability & Efficiency & Error rate & Stability & Efficiency & Error rate & Stability & Efficiency \\
\hline 500 & 0.11 & 97.9 & 98.3 & 0.35 & 73.4 & 79.1 & 0.31 & 70.4 & 80.1 \\
\hline 1000 & 0.11 & 97.6 & 98.5 & 0.32 & 72.9 & 78.6 & 0.36 & 73.9 & 81.6 \\
\hline 1500 & 0.11 & 97.5 & 98.4 & 0.31 & 71.8 & 78.1 & 0.31 & 71.8 & 79.1 \\
\hline 2000 & 0.12 & 97.4 & 98.7 & 0.32 & 70.9 & 77.9 & 0.32 & 72.9 & 75.9 \\
\hline 2500 & 0.12 & 97.3 & 97.2 & 0.35 & 69.8 & 77.7 & 0.38 & 75.8 & 80.7 \\
\hline 3000 & 0.12 & 97.1 & 97.8 & 0.36 & 69.3 & 77.5 & 0.36 & 79.8 & 80.5 \\
\hline 3500 & 0.13 & 96.9 & 97.2 & 0.37 & 69.1 & 77.1 & 0.37 & 61.9 & 80.2 \\
\hline
\end{tabular}

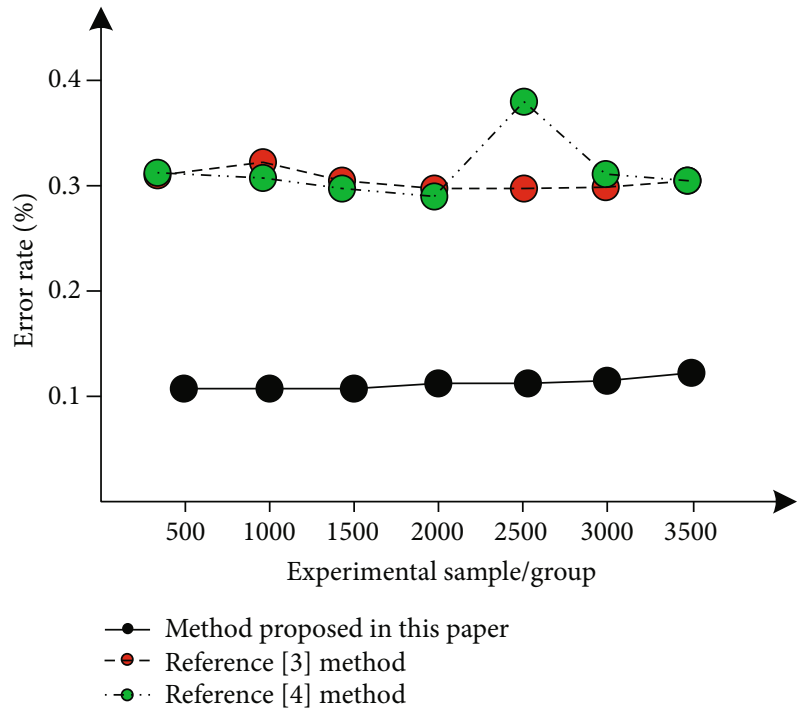

FIGURE 7: Comparison of error rates of different methods.

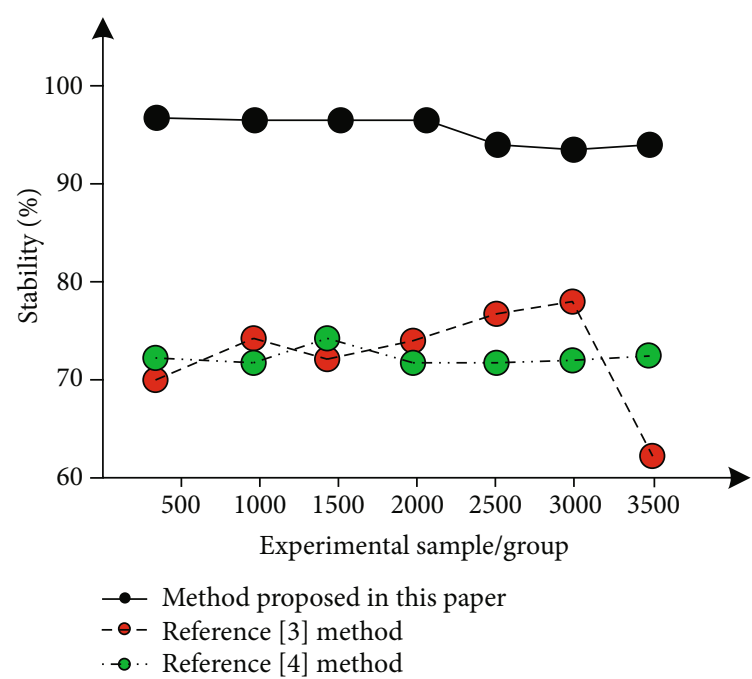

FIGURE 8: Stability comparison of different methods. matrix for optimizing and integrating teacher construction and grassroots work in the construction of grassroots teaching organizations, the concepts of node grassroots work entropy and two-dimensional grassroots work entropy in the cluster are introduced to describe the grassroots work of single-node data and overall teacher construction in the cluster in the construction of grassroots teaching organization. The particle swarm optimization algorithm $[22,23]$ is used to search the maximum value of grassroots work entropy, and this value is used to realize the intelligent automatic optimization of the fusion threshold of teacher construction and grassroots work in the construction of basic teaching organization and the intelligent optimization of organizational construction information is realized by using this value [24]. The specific steps are detailed below.

In the construction of grassroots teaching organizations, one-dimensional grassroots work entropy of sensor nodes represents the aggregation feature of the data distribution in the nodes, and the lower the one-dimensional entropy of sensor nodes is, the larger the range of the data arrangement of the nodes is (clustering feature is not obvious). Suppose $\{G, K\}$ represents the threshold of grass-roots work selection, in which $G$ represents the threshold of grassroots work, $K$ represents the threshold of grassroots work upper limit, $P$ represents the data proportion of the subset of teachers in the construction of grassroots teaching organizations, and the grassroots work entropy of single-node data in the construction of grassroots teaching organizations (average self-grass-roots work) is calculated by using the following formula:

$$
G(x)=\frac{p \cdot \ln p\{G, K\}}{(\bar{k} / k)^{\prime \prime}}
$$

In the process of optimizing and integrating the teacher construction and grassroots work in the construction of grassroots teaching organizations, $\max (G(x))$ represents the maximum value of $G(x)$, and $\left\{d_{n-\max }, d_{n-\min }\right\}$ represents the threshold value for selecting grassroots work data, and the two-dimensional grassroots work entropy of data is composed by introducing the characteristic quantity of spatial characteristics reflecting the distribution of grassroots teaching construction data, and the weighted average value 


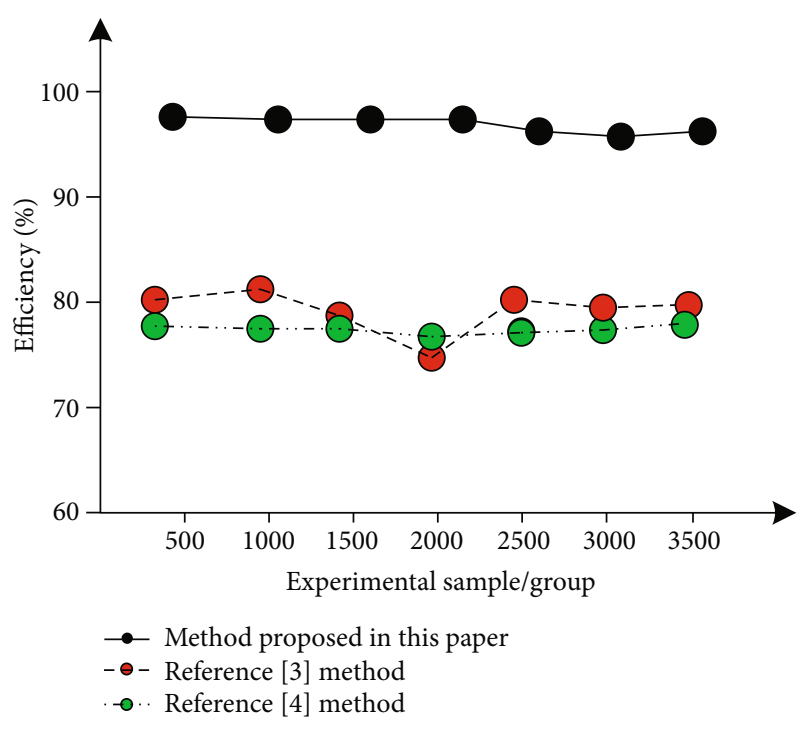

FIGURE 9: Time efficiency comparison of different methods.

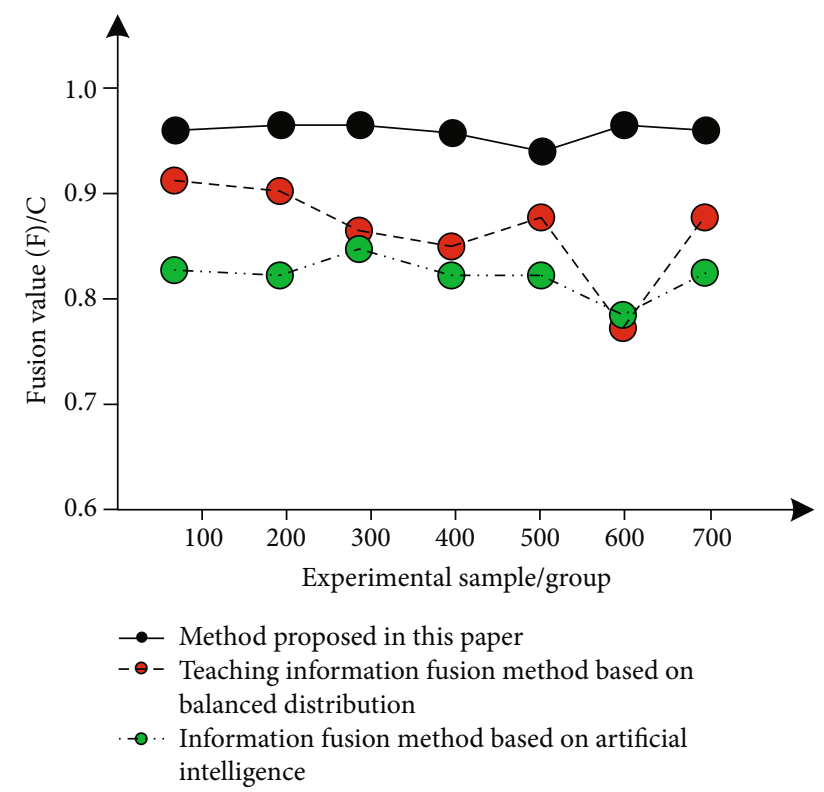

Figure 10: Comparison of information fusion values $(f)$ of different methods.

of intracluster data is defined as the characteristic quantity of spatial distribution characteristics of grassroots teaching construction data $[25,26]$.

Two-dimensional entropy in clusters where teacher development and grassroots work are integrated and discrete is defined as follows:

$$
G_{2}^{2}=\frac{f \cdot \ln \left(1-p_{i}\right)}{p}
$$

In the process of optimizing the integration of teacher construction and grassroots work in the construction of grassroots teaching organizations, assuming that $G_{0}$ repre-

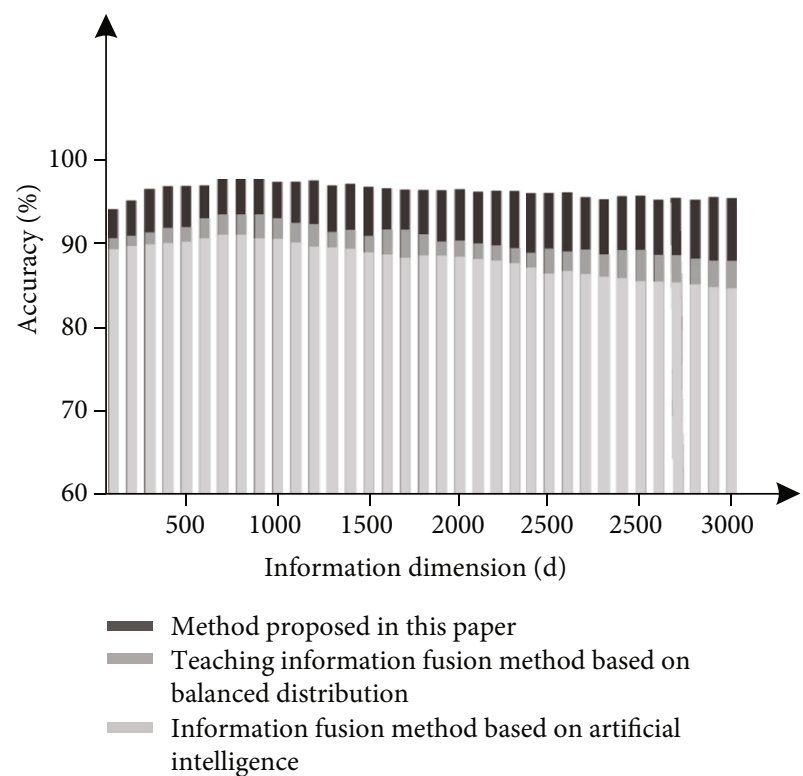

Figure 11: Comparison of test accuracy of different information fusion methods.

sents the threshold of two-dimensional entropy of teacher construction and grassroots work in the construction of grassroots teaching organizations, when the condition of $G_{2} \leq G_{0}$ is met, it can be concluded that there is an extreme data of abnormal changes in teacher construction in the construction of grassroots teaching organizations, and the integration of teacher construction and grassroots work in the construction of grassroots teaching organizations can be achieved by using $\{G, K\}$. When the teacher construction in the construction of grassroots teaching organizations has a tendency, the convergence of teacher construction and grassroots work in the construction of grassroots teaching organizations can be carried out according to the threshold $\{G, K\}$ between the upper and lower limits $[27,28]$. The particle swarm optimization algorithm is used to search for the maximum value of work entropy. The initial inertia factor is selected and expressed as follows:

$$
\Re(t+1)=\frac{\left(\Re_{\max }-\Re_{\min }\right)}{G_{2}^{2}} .
$$

In the above formula, $\mathfrak{R}_{\max }$ and $\mathfrak{R}_{\min }$, respectively, represent the maximum and minimum of the fusion weights of teacher construction and grassroots work in grassroots teaching organization construction.

The author makes use of the following methods to update the best adaptability of teacher building and grassroots work integration in grassroots teaching organization building:

$$
V_{i+1}=\frac{G}{\mathfrak{R}(t+1)} P
$$

Combining T-S fuzzy model with the BP neural network [29-31], the information fusion of basic teaching 
organization construction is realized. The specific process of information fusion using fuzzy neural network is as follows.

The T-S fuzzy structure has strong self-adaptive ability and has the characteristics of automatic update and fuzzy membership. According to the "if-then" rule, when the rule is $R^{j}$, the corresponding fuzzy reasoning rule is as follows [32-34]:

$$
\begin{gathered}
R^{j}: \text { If } x_{1} \text { is } A_{j}^{1}, x_{2} \text { is } A_{j}^{2}, \cdots, x_{M} \text { is } A_{j}^{m}, \\
\text { then } y_{j}=p_{j}^{0}+p_{j}^{1} x_{1}+\cdots+p_{j}^{m} x_{m} .
\end{gathered}
$$

In particular, $A_{j}^{i}$ represents the fuzzy set of information on the platform, $p_{j}^{i}(i=1,2, \cdots, m)$ represents the platform parameters (weights), and $y_{j}$ represents the output of fuzzy rules. The input information is ambiguous and the output is unambiguous [35].

There are five layers in the fuzzy neural network, which are the input layer, fuzzy layer, fuzzy rule layer, antifuzzy layer, and output layer. The number of nodes in the input layer is the same as the $x_{i}$ dimension of the input variable. After being processed by the fuzzy layer, the output membership $u_{j}^{i}$ is fuzzy calculated through the fuzzy rule layer $\omega^{k}$, and the final output value is calculated by the formula (13) [36].

In the process of multiplatform information fusion using the fuzzy neural network, the formula for calculating the error $\varepsilon$ between output value and expected value is as follows:

$$
\varepsilon=\frac{(\chi-E)^{2}}{2}
$$

Here, $\varepsilon$ represents the actual output and $E$ represents the expected value. According to the expected error value, the output result is modified adaptively.

The weights between the first and the second, the second and the third layers of the FNN are set to 1, the membership function parameters and the network weight of the fourth layer are modified through the learning process; The information fusion of grassroots teaching organization construction is realized by using the modified fuzzy neural network, and the information fusion of the FNN is realized.

\section{Countermeasures}

(1) Perfecting and implementing the institutionalized work system is a necessary guarantee for the order of teaching and research work, a method for making the system of grassroots teaching organizations complete and standardized and a basic norm for promoting the harmony and stability of grassroots organizations. Taking an institutionalized approach, the fate of the members of the organization will be linked with the rise and fall of the organization and help to strengthen the collective cohesion. The teaching and research section of colleges and universities is a grassroots work organization, and the relevant work is completed within the framework of schools and colleges. The system of the teaching and research section is used to ensure the orderly development of the basic work of teaching and research, including the system of teaching and research activities, the system of class evaluation, the system of teaching inspection, and the system of filing materials

(2) Selecting the person in charge of the teaching and research section

The director of grassroots teaching and research department plays an important role in cultivating students, improving teaching level, and cultivating talents. Their specific duties include completing the tasks of teaching, scientific research, and team building assigned by their superiors on time and in good quality and in good quantity; coordinating and organizing the professional construction, curriculum construction, and textbook construction of their own rooms; studying, enriching, and renewing the teaching contents, reforming the teaching means and methods, and continuously improve the teaching quality; we will carry out the construction of teaching staff and do a good job of teaching, helping, and guiding old, middle-aged and young teachers, and doing a good job in the work of helping and guiding the old, middle, and young teachers; and doing a good job in the examination and management of teachers. In view of the above responsibilities, it is necessary to do a good job of selecting and training candidates for the teaching and research section. Specifically, in terms of teaching ability, the director of the teaching and research department must have a strong understanding of the importance of teaching and teaching skills; in terms of management ability, he must be able to handle complicated and trivial daily affairs, so as to avoid the inefficient situation reported in detail; in terms of academic level, he must be able to lead a team to do a good job in teaching and research, complete the selection of teaching and research topics, and write a declaration. The comprehensive ability request needs to carry on the selective training for the target candidate; the investigation study and the exchange discussion are the essential person in charge raises the method.

(3) Optimize the structure of the teaching staff

The overall quality of teachers is the key to doing a good job in personnel training. Whether its structure is reasonable or not will affect the level of full-time teachers and school quality. Firstly, talent introduction is a direct way to optimize the structure of teachers. According to the needs of professional construction, high-level talents with relevant knowledge structure are introduced to improve the overall strength of teaching team. Secondly, it is very important to do a good job in the training of internal teachers. While introducing fresh blood, we need to fully contact the professional expertise and direction of existing teachers, and do a good job in the reconstruction of the corresponding teaching team, so as to improve their hematopoietic function. In terms of teaching staff facilities, the training mechanism of 
"please come in and send out" should be actively promoted. Specifically, through a variety of platforms, we can invite experienced scholars to exchange guidance and invite people with industry skills to give lectures in order to improve teachers' knowledge structure and industry awareness. At the same time, actively encourage frontline teachers to participate in teaching and research meetings to carry out teaching and learning, to promote teaching and research capacity.

(4) Strengthen the construction of teaching and scientific research teams

The construction of the teaching and research team is the basis of improving the overall level of teaching and research department. Taking curriculum construction as the core, reforming teaching means, optimizing teaching methods, and content are the core goals of team building. First, teachers should be guided to take the curriculum teaching reform at the point, fully analyze the insufficiency in the current teaching work, and do a good job of teaching communication and teaching reform. Teachers, textbooks, and students should form a good interactive teaching community, fully interact with each other, improve the current teaching situation, and improve the teaching level. Second, take scientific research as the core to promote the integration of teaching teams. The teaching staff of applied undergraduate colleges need to be "indomitable," not only have their own front research sensitivity and tracking ability but also have the ability to combine technology with industry production. Through the corresponding scientific research exchange, enrich the existing teaching content in order to improve the teaching quality. It is an effective way to promote scientific research exchange or teaching discussion in a planned way.

\section{Experimental Results and Analysis}

In order to verify the comprehensive effectiveness of the proposed information fusion method for the construction of grassroots teaching organizations based on intelligent optimization, simulation experiments need to be designed. The specific experimental environment is Dell PC, the processor type is Intel Core M480I5, the main frequency is $2.67 \mathrm{GHz}$, the memory is $4 \mathrm{~GB}$, and the operating framework of Windows 10 and 32 bits is Labmat2020.

5.1. Energy Consumption and Accuracy Measurement. In the course of the experiment, the node energy of the spatial data in the grassroots teaching organization construction network is $1 \mathrm{~J}$, and the converged node energy is $90 \mathrm{~J}$. The random generation of all the nodes in the grassroots teaching organization construction network is distributed in the grid area of $200 \times 200$. The spatial data nodes are located in the center of the region, rotational period is $30 \mathrm{~s}$, experimental time is $300 \mathrm{~s}$, and the wireless communication distance is $200 \mathrm{~m}$. The information fusion experiment of the grassroots teaching organization construction network is carried out, respectively, by using the method of teaching information fusion based on balanced distribution, the method of infor- mation fusion based on artificial intelligence proposed in the reference [3], the method of information fusion based on artificial intelligence proposed in the reference [4], and the method presented in this paper. The energy consumption and accuracy of information fusion are compared with two different algorithms. The comparison results are shown in Figures 5 and 6.

As can be seen from Figure 5, the energy consumption of the proposed method is significantly lower than that of the comparison method, and the maximum energy consumption is $0.7 \mathrm{~J}$. It can be seen from Figure 6 that the accuracy of using the proposed method to integrate the construction information of grassroots teaching organizations is higher than $0.93 \%$, which is higher than that of the comparative method. Therefore, using the improved method for information fusion not only ensures the effectiveness of data fusion but also reduces the communication flow and not only reduces the average energy consumption but also significantly improves the accuracy of information fusion.

5.2. Comparative Test of Error Rate, Stability, and Time Efficiency. At different experimental times, the teaching information fusion method based on balanced distribution proposed by this method and reference [3] and the information fusion method based on artificial intelligence proposed by reference [4] are used for information fusion experiments. The test indexes are error rate, stability, and efficiency. Based on the results of comparison, the comprehensive effectiveness of information fusion is measured.

In order to more intuitively analyze the comparison of error rate, stability, and time efficiency indexes of different methods, the data in Table 1 are drawn into simulation diagrams, as shown in Figure 7-9.

The experimental results show that the comprehensive effectiveness of using the improved method to fuse spatial data and grassroots teaching construction information in grassroots teaching construction sensor network is remarkable. This is mainly because the improved algorithm uses the particle swarm optimization algorithm to search the maximum value of grassroots teaching construction information entropy and realize the automatic optimization of grassroots teaching construction information fusion threshold. Thus, it ensures the comprehensive effectiveness of the integration of spatial data and information in the grassroots teaching construction sensor network, reduces energy consumption, and prolongs the service life of the grassroots teaching construction sensor network.

5.3. Test of Fusion Value Index. The proposed information fusion method is used for experiments with the teaching information fusion method based on balanced distribution proposed in reference [3] and the information fusion method based on artificial intelligence proposed in reference [4], and the corresponding information fusion values of the three methods are compared. The experimental results are shown in Figure 10. The organization of information fusion value $f$ was set as $C$. 
According to Figure 10, when the experimental samples are different, the information fusion values of the three methods change within a certain range, and the curve of the information fusion value of the three methods has the least fluctuation, which shows that the method has strong adaptability and high recognition degree for all kinds of information; when the experimental samples are the same, the information fusion values of the three methods have the greatest and the best information fusion effect.

5.4. Comparative Test of Fusion Accuracy under Different Information Output Dimensions. Comparing the test accuracy of three different methods with different information output dimensions, the experimental results are shown in Figure 11.

According to Figure 11, with the increase of information output dimension, the test accuracy of the other two information fusion methods in the experimental comparison shows a downward trend, and the test accuracy of the proposed method changes very little. The above experimental results show that the convergence speed and classification accuracy of the proposed information fusion method are better than the experimental comparison method.

\section{Conclusion}

In order to solve the problems that the information processing accuracy of traditional teaching construction information fusion methods is low and the test results of information fusion indicators are not ideal, a new information fusion method for grassroots teaching organization construction based on intelligent optimization is proposed in this paper. Experiments show that the error rate, stability, and efficiency of the proposed method are high, and the information fusion value is more than 0.95 . The fusion accuracy under different information output dimensions is about 93\% 98\%. The experimental results show that the application performance of the proposed method is better. However, although the particle swarm optimization algorithm can comprehensively deal with the construction information of grassroots teaching organization, it is easy to fall into the local optimal solution. Therefore, this will be the next research direction of this paper to further improve the information fusion efficiency of grassroots teaching organization construction.

\section{Data Availability}

The raw data supporting the conclusions of this article will be made available by the authors, without undue reservation.

\section{Conflicts of Interest}

The author declared that they have no conflicts of interest regarding this work.

\section{References}

[1] E. Giannopoulou, P. J. Barlatier, and J. Pénin, "Same but different? Research and technology organizations, universities and the innovation activities of firms," Research Policy, vol. 48, no. 1, pp. 223-233, 2019.

[2] X. R. Wu, B. Q. Liu, and T. T. Yuan, "A new generation of smart class: concept, platform and system architecture," China Educational Technology, vol. 3, pp. 81-88, 2019.

[3] J. Zhu and X. H. Lu, "Multimedia integrated learning resources information integration system in cloud storage environment," Information Technology, vol. 43, no. 6, p. 5, 2019.

[4] B. C. Pan, "Design and implementation of intelligence classroom platform based on artificial intelligence," Heilongjiang Science, vol. 11, no. 23, pp. 8-9, 2020.

[5] L. Zhao, "Fast fusion simulation of distributed multi-source information in network video," Computer Simulation, vol. 37, no. 6, pp. 173-176, 2020.

[6] M. D. Abdulrahaman, N. Faruk, A. A. Oloyede et al., "Multimedia tools in the teaching and learning processes: a systematic review," Heliyon, vol. 6, no. 11, 2020.

[7] H. Tanveer, T. Balz, N. S. Sumari, R. U. Shan, and H. Tanweer, "Pattern analysis of substandard and inadequate distribution of educational resources in urban-rural areas of Abbottabad, Pakistan," Geo Journal, vol. 85, no. 5, pp. 1397-1409, 2020.

[8] T. L. Friesz, "Socio-economic determinants of student mobility and inequality of access to higher education in Italy," Networks and Spatial Economics, vol. 19, no. 1, pp. 125-148, 2019.

[9] D. Y. Jiang and University H H, "Exploration on the reform of database series course system in colleges and universities based on the training of applied talents," Heilongjiang Science, vol. 10, no. 9, pp. 102-103, 2019.

[10] Q. Xia, G. Liu, and D. Ma, "Research on innovative talents training mode in local colleges and universities meeting the needs of regional economic development," Shandong Chemical Industry, vol. 48, no. 19, pp. 219-220, 2019.

[11] S. Salcedo-Sanz, P. Ghamisi, M. Piles et al., "Machine learning information fusion in Earth observation: a comprehensive review of methods, applications and data sources," Information Fusion, vol. 63, pp. 256-272, 2020.

[12] L. Huang, Z. Liu, Q. Pan, and J. Dezert, "Evidential combination of augmented multi-source of information based on domain adaptation," SCIENCE CHINA Information Sciences, vol. 63, no. 11, pp. 1-14, 2020.

[13] X. Guan, J. Zhao, and H. Q. Liu, “A new basic probability assignment method for multi-source and high conflict information and its application," in Materials Science Forum, vol. 960, pp. 100-104, Trans Tech Publications Ltd, 2019.

[14] M. Z. Iqbal, D. Bobkov, and E. Steinbach, "Adaptive fusionbased 3D keypoint detection for RGB point clouds," in 2019 IEEE International Conference on Image Processing (ICIP), Taipei, Taiwan, 2019.

[15] X. Wang, L. Wang, and Y. Hao, "A construction of multisender authentication codes from eigenvalues and eigenvectors of the matrix over finite fields," Journal of Harbin Institute of Technology (New Series), vol. 26, no. 1, pp. 51-60, 2019.

[16] V. S. Cotronei-Baird, "Academic hindrances in the integration of employability skills development in teaching and assessment practice," Higher Education: The International Journal of Higher Education Research, vol. 79, no. 2, pp. 203-223, 2020.

[17] A. Gee and H. Abbass, "Transparent machine education of neural networks for swarm shepherding using curriculum design," in 2019 International Joint Conference on Neural Networks (IJCNN), vol. 67no. 13, pp. 57-69, Budapest, Hungary, 2019. 
[18] G. R. Browne, H. Bender, J. Bradley, and A. Pang, "Evaluation of a tertiary sustainability experiential learning program," International Journal of Sustainability in Higher Education, vol. 21, no. 4, pp. 699-715, 2020.

[19] A. Ghosh, R. Saharay, S. Chakrabarty, and S. Bhadra, "Robust generalised quadratic discriminant analysis," Pattern Recognition, vol. 117, no. 6, p. 107981, 2021.

[20] T. Chen, Q. Zhu, M. X. Zhou, and S. Wang, "Trust-based recommendation algorithm in social network," Journal of Software, vol. 28, no. 3, pp. 721-731, 2017.

[21] W. Zhang and Z. J. Wang, "Research on join operation of temporal big data in distributed environment," Computer Engineering, vol. 45, no. 3, 2019.

[22] M. A. Akram, P. Liu, M. O. Tahir, W. Ali, and Y. Wang, "A state optimization model based on Kalman filtering and robust estimation theory for fusion of multi-source information in highly non-linear systems," Sensors, vol. 19, no. 7, p. 1687, 2019.

[23] A. Khodar, L. V. Chernenkaya, I. Alkhayat, H. A. F. Al-Afare, and E. N. Desyatirikova, "Design model to improve task scheduling in cloud computing based on particle swarm optimization," in 2020 IEEE Conference of Russian Young Researchers in Electrical and Electronic Engineering (EIConRus), St. Petersburg and Moscow, Russia, 2020.

[24] N. Bilandi, H. K. Verma, and R. Dhir, "hPSO-SA: hybrid particle swarm optimization-simulated annealing algorithm for relay node selection in wireless body area networks," Applied Intelligence, vol. 51, pp. 1410-1438, 2021.

[25] Y. Ebadi and N. J. Navimipour, “An energy-aware method for data replication in the cloud environments using a Tabu search and particle swarm optimization algorithm," Concurrency \& Computation Practice \& Experience, vol. 31, no. 1, 2019.

[26] P. Li, "Node localization method for massive sensor networks based on clustering particle swarm optimization in cloud computing environment," International Journal of Wavelets Multiresolution \& Information Processing, vol. 18, no. 1, pp. 66-77, 2020.

[27] F. N. Robati and S. Iranmanesh, "Inflation rate modeling: adaptive neuro-fuzzy inference system approach and particle swarm optimization algorithm (ANFIS-PSO)," MethodsX, vol. 7, no. 1, p. 101062, 2020.

[28] R. M. Adnan, R. R. Mostafa, O. Kisi, Z. M. Yaseen, S. Shahid, and M. Zounemat-Kermani, "Improving streamflow prediction using a new hybrid ELM model combined with hybrid particle swarm optimization and grey wolf optimization," Knowledge-Based Systems, vol. 230, no. 6, p. 107379, 2021.

[29] M. S. Ali, G. Narayanan, V. Shekher, H. Alsulami, and T. Saeed, "Dynamic stability analysis of stochastic fractionalorder memristor fuzzy BAM neural networks with delay and leakage terms," Applied Mathematics and Computation, vol. 369, p. 124896, 2020.

[30] M. S. Ali, G. Narayanan, S. Saroha, B. Priya, and G. K. Thakur, "Finite-time stability analysis of fractional-order memristive fuzzy cellular neural networks with time delay and leakage term," Mathematics and Computers in Simulation, vol. 185, pp. $468-485,2021$.

[31] R. Naderkhani, M. H. Behzad, T. Razzaghnia, and R. Farnoosh, "Fuzzy regression analysis based on fuzzy neural networks using trapezoidal data," International Journal of Fuzzy Systems, vol. 23, no. 5, pp. 1267-1280, 2021.
[32] N. Guo, Y. Fang, Z. Tian, and S. Cao, "Research on SOC fuzzy weighted algorithm based on GA-BP neural network and ampere integral method," Journal of Engineering, vol. 2019, no. 15, 580 pages, 2019.

[33] T. Mandhula, S. Pabboju, and N. Gugulotu, "Predicting the customer's opinion on amazon products using selective memory architecture-based convolutional neural network," The Journal of Supercomputing, vol. 76, no. 8, pp. 5923-5947, 2020.

[34] T. H. Sardar and Z. Ansari, "Distributed big data clustering using map reduce-based fuzzy C-medoids," Journal of The Institution of Engineers (India): Series B, vol. 103, pp. 73-82, 2022.

[35] C. Zhang, S. K. Oh, and Z. Fu, "Hierarchical polynomial-based fuzzy neural networks driven with the aid of hybrid network architecture and ranking-based neuron selection strategies," Applied Soft Computing, vol. 113, article 107865, 2021.

[36] V. T. Yen, W. Y. Nan, and P. V. Cuong, "Recurrent fuzzy wavelet neural networks based on robust adaptive sliding mode control for industrial robot manipulators," Neural Computing and Applications, vol. 31, no. 11, pp. 6945-6958, 2019. 